\title{
Outbreak of cephalosporin resistant Enterobacter cloacae infection in a neonatal intensive care unit
}

\author{
N MODI, V DAMJANOVIC, AND R W I COOKE \\ Department of Child Health, Liverpool Maternity Hospital, University of Liverpool, and Department of \\ Microbiology, Sefton General Hospital, Liverpool
}

SUMMARY Enterobacter cloacae resistant to third generation cephalosporins emerged rapidly during an outbreak of serious infections due to this organism in a neonatal intensive care unit where ampicillin and gentamicin were used as first line antibiotic treatment. Organisms resistant to cephalosporins were isolated from 12 infants, six of whom developed systemic infection. Two infants died. Isolates of $E$. cloacae from four of five infants treated with cefotaxime showed a loss of sensitivity to this antibiotic during treatment, but in the three infants who survived sensitive organisms were again isolated after treatment had stopped. Stopping treatment with the cephalosporins, closure of the unit to new admissions, and strict cohorting of colonised infants resulted in a prompt end to the outbreak.

This outbreak suggests that the routine use of third generation cephalosporins for suspected sepsis may be inappropriate in the presence of a large reservoir of organisms with the potential for rapidly developing resistance. Routine bacteriological surveillance, however, might permit their use on a rotational basis.

Infection is common in babies in neonatal intensive care units. It may be overwhelming but early signs are largely non-specific and babies from such a population often receive broad range antibiotics before the results of cultures are available. The combination of an aminoglycoside and a penicillin is widely used. ${ }^{1}$ Recently, the emergence of gentamicin resistant organisms and the potential problems of aminoglycoside ototoxicity, nephrotoxicity, and poor penetration into cerebrospinal fluid (CSF) has led to third generation cephalosporin monotherapy being advocated for routine first line treatment. ${ }^{2} \mathrm{We}$ describe our experience of third generation cephalosporins in a unit that has continued to use ampicillin and gentamicin as first line treatment. We suggest that the routine use of third generation cephalosporins as first line treatment for suspected infection in neonatal intensive care units may be inappropriate.

\section{Background}

The regional neonatal intensive care unit, Liverpool Maternity Hospital, admits between 450 and 500 babies each year. Roughly $30 \%$ weigh less than $1500 \mathrm{~g}$ at birth and about $30 \%$ are outborn.
Handwashing with a chlorhexidine solution followed by application of an alcohol rub is carried out before every infant contact. Gloves are worn during nursing procedures and napkin changes. Routine infection surveillance includes culture and sensitivity testing on daily specimens of tracheal aspirate from intubated babies and weekly rectal and superficial swabs from all babies. A full infection screen, consisting of superficial swabbing and urine, CSF, and blood cultures, is carried out on all outborn babies on admission.

The combination of ampicillin and gentamicin has been used as the standard initial treatment of suspected infection for five years. Over $95 \%$ of babies receive broad range antibiotics at least once during their admission.

\section{Laboratory methods}

Standard methods and media were used for isolation of organisms. ${ }^{3}$ Species identification of isolates was carried out on the basis of biochemical reactions, using the API 20E system (API Products, Basingstoke). Sensitivities to selected antibiotics were determined by the breakpoint method, using Oxoid DST agar and a multipoint inoculator that produced 
an inoculum of $10^{5}$ colony forming units. To incorporate antibiotics of required concentrations into the DST agar, Adatabs (Mast House, Bootle) were used. Breakpoints were $1 \mathrm{mg} / 1$ (low) and $4 \mathrm{mg} / 1$ (high) for gentamicin and $2 \mathrm{mg} / \mathrm{l}$ (low) and $8 \mathrm{mg} / \mathrm{l}$ (high) for cephalosporins. The plates were incubated at $37^{\circ} \mathrm{C}$ overnight. Isolates were considered resistant to gentamicin or to the tested cephalosporin if growth was noticed on plates containing $4 \mathrm{mg} / \mathrm{l}$ or $8 \mathrm{mg} / \mathrm{l}$ of respective antibiotic.

\section{Clinical observations}

The pattern of colonisation in this unit is one of fluctuating numbers of infants colonised with Staphylococcus epidermidis, yeasts, and strains of Pseudomonas, Klebsiella, Serratia, and Enterobacter.

From March 1985 increasing numbers of isolates of gentamicin resistant Staph. epidermidis resulted in the use of cefuroxime for infections caused by this organism. Infection with Staph. epidermidis accounts for most blood cultures that produce positive results and cefuroxime therefore became the third most commonly used antibiotic in the unit. Concurrent with this increase in use of cefuroxime routine bacteriological surveillance revealed an increasing proportion of babies colonised with Enterobacter cloacae, most of whom were resistant to cefuroxime. Up to the end of June 1985 all isolates of $E$. cloacae were sensitive to the third generation cephalosporins cefotaxime and ceftazidime. During July strains resistant to all cephalosporins tested first occurred.

Results of weekly bacteriological surveillance from 4 June 1985 to 17 September 1985 are presented in the Figure. Screening swabs were obtained from each baby on the unit every Tuesday. The total number of babies in the unit is shown with the proportion colonised with E. cloacae and the proportion harbouring strains resistant to cefotaxime. Resistant organisms were recovered from 12 infants on 21 occasions over an eight week period. Seven of these infants had not been on treatment with a cephalosporin at the time of isolation of the resistant strain.

During this time a cluster of serious infections with $E$. cloacae arose, with two episodes of ventriculitis and septicaemia (one episode of ventriculitis prolonged at 19 days) and four more cases of isolated septicaemia. Two infants died and two required treatment with the new quinolone ciprofloxacin. The isolates of $E$. cloacae from five of these six infants with systemic infection were initially sensitive to cefotaxime, and these five were thus treated with cefotaxime: isolates of $E$. cloacae

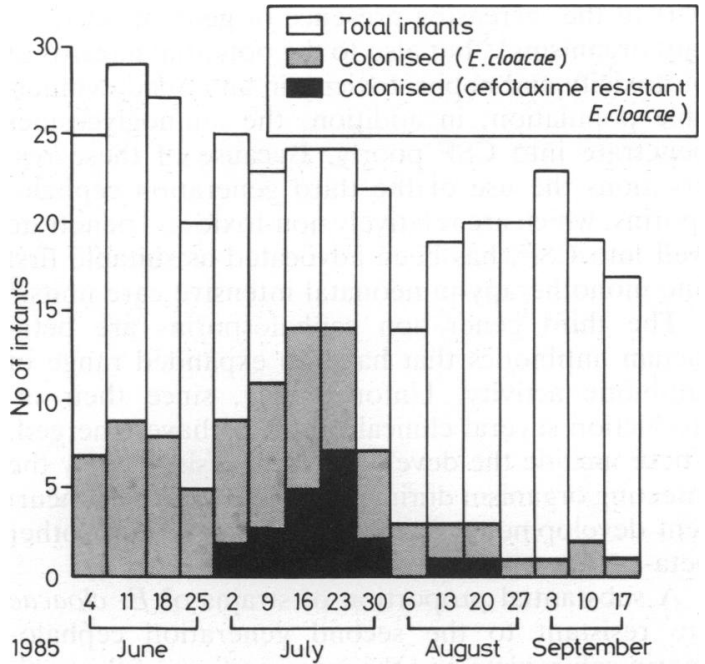

Figure Total No of babies on the neonatal unit by weekly intervals on the days that infection screening was undertaken, showing the proportion colonised with Enterobacter cloacae and with cefotaxime resistant strains.

Table Details of infants with systemic Enterobacter cloacae infection initially sensitive to cefotaxime but who developed resistance after treatment

\begin{tabular}{lllll}
\hline $\begin{array}{l}\text { Case } \\
\text { No }\end{array}$ & $\begin{array}{l}\text { Birth weight } \\
(\mathrm{g})\end{array}$ & $\begin{array}{l}\text { Gestational } \\
\text { age } \\
\text { (weeks) }\end{array}$ & Infection & Days* \\
\hline 1 & 1840 & 30 & BCs & 7 \\
2 & 1210 & 28 & BCs, CSF & 7 \\
3 & 1090 & 28 & BCs & 2 \\
4 & 1320 & 27 & BCs, CSF & 3 \\
\hline
\end{tabular}

${ }^{*}$ Days after start of treatment that resistant organisms isolated. $\mathrm{BCs}=$ Blood cultures; $\mathbf{C S F}=$ Cerebrospinal fluid.

from four revealed a loss of sensitivity to cefotaxime and to other third generation cephalosporins during treatment (Table). In the three of these four infants who survived $E$. cloacae sensitive to the third generation cephalosporins was once again isolated after exposure to cefotaxime had ceased.

Infection control measures were begun and included strict cohorting of infants colonised with $E$. cloacae, stopping treatment with all cephalosporins, and closure of the unit to new admissions. Third generation resistant strains of $E$. cloacae disappeared promptly and the level of colonisation with this organism fell rapidly.

\section{Discussion}

Concern has been voiced regarding the use of gentamicin in neonatal intensive care units due in 
part to the increasing presence of gentamicin resistant organisms ${ }^{45}$ but also to the potential hazards of ototoxicity and nephrotoxicity in an already vulnerable population; in addition, the aminoglycosides penetrate into CSF poorly. Because of these reservations the use of the third generation cephalosporins, which are relatively non-toxic and penetrate well into CSF, has been advocated as suitable first line monotherapy in neonatal intensive care units. ${ }^{2}$

The third generation cephalosporins are betalactam antibiotics that have an expanded range of antibiotic activity. Unfortunately, since their introduction several clinical problems have emerged. These include the development of resistance by the infecting organism during treatment and the concurrent development of cross resistance to most other beta-lactam antibiotics. ${ }^{6} 7$

A substantial proportion of strains of $E$. cloacae are resistant to the second generation cephalosporin cefuroxime and the increased use of this antibiotic for Staph. epidermidis infections was undoubtedly responsible for the selection of such resistant strains, with a consequent increase in numbers of infants colonised with $E$. cloacae. This enlarging reservoir of $E$. cloacae thus consisted of organisms resistant to cefuroxime but sensitive to the third generation cephalosporins cefotaxime and ceftazidime. Concurrent with the increasing number of infants colonised with $E$. cloacae, however, strains resistant to cefotaxime and ceftazidime occurred.

The possibility of introduction of strains of $E$. cloacae resistant to the third generation cephalosporins from outside the unit was unlikely as all outborn babies underwent a full infection screen on admission. Furthermore, the biotypes of the isolates were identical.

Production of beta-lactamase activity may be either plasmid or chromosomally mediated; the oxime cephalosporins, cefotaxime and ceftazidime, show a high degree of stability to plasmid encoded beta-lactamase activities and are also relatively resistant to the normal amounts of chromosomally mediated cephalosporinase activities found in most wild type Enterobacteriacae. ${ }^{8}$ If these amounts are increased, however, either by exposure of the wild type to a beta-lactamase inducer (reversible derepression) or mutation to the stably derepressed state, most third generation cephalosporins may be inactivated. ${ }^{910}$ Both second and third generation cephalosporins are inducers of cephalosporinase activities in the genera Pseudomonas, Serratia, and Enterobacter, which possess chromosomally mediated inducible beta-lactamase activities (type $\left.\mathrm{I}^{11}\right){ }^{7}$

Both mechanisms of increased production of beta-lactamase activity may have played a part in the outbreak of infection described. E. cloacae resistant to the third generation cephalosporins were isolated from seven babies on routine screening swabs. At the time of isolation none had been on treatment with a cephalosporin. This observation suggests nosocomial transmission, although this cannot be proved as no information regarding serotypes was available. The biotypes of the isolates were, however, identical. As induction requires the continuing presence of the inducer, with induction ceasing when the inducer is removed, induction of beta-lactamase activity was probably not involved here; the passage of a derepressed mutant is a possibility.

In four of the five infants with systemic infection treated with cefotaxime $E$. cloacae resistant to cefotaxime and cefotazidime were first isolated after the start of treatment (Table). Once again, though the possibility of multiple strains exists, reversible induction of production of beta-lactamase activity was probably responsible for the occurrence of resistance here. This is supported by the observation that in the three infants who survived $E$. cloacae isolated after exposure to cefotaxime had ceased was again sensitive to the third generation cephalosporins.

The emergence of cefotaxime resistance during treatment with this antibiotic is recognised ${ }^{5712}$ but has been described only once before in a neonatal intensive care unit. ${ }^{5}$ This problem is compounded because multiple drug resistance may arise simultaneously. Emergence of beta-lactam resistance during treatment with a combination of a third generation cephalosporin and an aminoglycoside has also been reported. ${ }^{13}$

Strains possessing inducible lactamase activities from the genera Pseudomonas, Serratia, and Enterobacter are commonly responsible for colonisation of infants in neonatal intensive care units. E. cloacae has been shown to have a high mutation rate. ${ }^{10}$ The common need for treatment with antibiotics, the predilection of these infants to nosocomial infection, and the rapid emergence of resistance to the third generation cephalosporins, such resistance additionally occurring during treatment, suggests that the routine use of these agents is inappropriate in the presence of a large reservoir of infants colonised with the above organisms. First line monotherapy with a third generation cephalosporin may well, however, be appropriate in the absence of such a pool of organisms. The value of the third generation cephalosporins for isolated, proven infection is undisputed. The epidemiological considerations governing the frequent use of an antibiotic blind as first line treatment for suspected sepsis must be clearly distinguished, however, from the use of an 
antibiotic relatively infrequently for proven sepsis. An antibiotic suitable in one context may not be suitable in the other.

Our observations once again illustrate the importance of routine bacteriological surveillance in a neonatal intensive care unit. ${ }^{14}$ Routine surveillance also permits the introduction of alternative first line treatment regimens on a rotational basis.

Our thanks to Mrs Dorothy Bolger for typing the manuscript, to Mr Ken Walters for providing the illustration, and to Professor C A Hart for his advice.

\section{References}

1 Roberton NRC. A manual of neonatal intensive care. 2nd ed. London: Edward Arnold, 1986.

${ }^{2}$ De Louvois J. Serious infections in the newborn. Serious Infections Update, 1985: No 7.

${ }^{3}$ Stokes EJ, Ridgeway GL. Clinical bacteriology. 5th ed. London: Edward Arnold, 1980.

${ }^{4}$ Anderson EL, Heiber JP. An outbreak of gentamicin resistant E. cloacae infection in a paediatric intensive care unit. Infect Control 1983;4:148-52.

5 Bryan CS, John JF, Pai S, Austin TL. Gentamicin v cefotaxime for therapy of neonatal sepsis: relationship to drug resistance. Am J Dis Child 1985;139:1086-9.

${ }^{6}$ Sanders CC, Sanders WE. Microbial resistance to newer generation $\beta$ lactam antibiotics: clinical and laboratory implications. J Infect Dis 1985;151:399-406.
${ }^{7}$ Sanders CC, Sanders WE. Emergence of resistance during therapy with the newer $\beta$ lactam antibiotics. Role of inducible $\beta$ lactamases and implications for the future. Rev Infect Dis 1983;5:639-48.

${ }^{8}$ Sykes RB, Bush K. Interaction of new cephalosporins with $\beta$ lactamases and $\beta$ lactamase producing Gram negative bacilli. Rev Infect Dis 1983;5(Suppl 2):356-67.

${ }^{9}$ Collatz E, Gutmann L, Williamson R, Azar J. Development of resistance to $\beta$ lactam antibiotics with special reference to third generation cephalosporins. J Antimicrob Chemother 1984;14 (Suppl B): 13-21.

10 Seeberg AH, Tolxdorff-Neutzling RM, Wiedemann B. Chromosomal $\beta$ lactamases of $E$. cloacae are responsible for resistance to third generation cephalosporins. Antimicrob Agents Chemother 1983;23:918-25.

1 Richmond MN, Sykes RB. The $\beta$ lactamases of Gram negative bacteria and their possible physiological role. $A d v$ Microb Physiol 1973;9:31-8.

12 Karakusis PM, Feczko JM, Goodman LJ, et al. Clinical efficacy of cefotaxime in serious infections. Antimicrob Agents Chemother 1982;21:119-24.

13 King A, Shannon K, Eykyn S, Phillips I. Reduced sensitivity to $\beta$ lactam antibiotics arising during ceftazidime treatment of Pseudomonas acruginosa infections. J Antimicrob Chemother 1983;12:363-70.

${ }^{14}$ Morgan MEI, Hart CA, Cooke RWI. Klebsiella infection in a neonatal intensive care unit: role of bacteriological surveillance. J Hosp Infect 1984;5:377-85.

Correspondence to Dr N Modi, Department of Child Health, Alder Hey Children's Hospital, Eaton Road, Liverpool L12 2AP, England.

Received 15 August 1986 